\title{
Review Article \\ Nrf2 Signaling and the Slowed Aging Phenotype: Evidence from Long-Lived Models
}

\author{
Danielle R. Bruns, Joshua C. Drake, Laurie M. Biela, Frederick F. Peelor III, \\ Benjamin F. Miller, and Karyn L. Hamilton \\ Department of Health and Exercise Science, Colorado State University, 220 Moby B Complex, Fort Collins, CO 80523-1582, USA \\ Correspondence should be addressed to Danielle R. Bruns; danielle.bruns@ucdenver.edu
}

Received 8 April 2015; Revised 28 May 2015; Accepted 4 June 2015

Academic Editor: Claudio Cabello-Verrugio

Copyright (c) 2015 Danielle R. Bruns et al. This is an open access article distributed under the Creative Commons Attribution License, which permits unrestricted use, distribution, and reproduction in any medium, provided the original work is properly cited.

\begin{abstract}
Studying long-lived animals provides novel insight into shared characteristics of aging and represents a unique model to elucidate approaches to prevent chronic disease. Oxidant stress underlies many chronic diseases and resistance to stress is a potential mechanism governing slowed aging. The transcription factor nuclear factor (erythroid-derived 2)-like 2 is the "master regulator" of cellular antioxidant defenses. Nrf2 is upregulated by some longevity promoting interventions and may play a role in regulating species longevity. However, Nrf2 expression and activity in long-lived models have not been well described. Here, we review evidence for altered Nrf2 signaling in a variety of slowed aging models that accomplish lifespan extension via pharmacological, nutritional, evolutionary, genetic, and presumably epigenetic means.
\end{abstract}

\section{Introduction}

The incidence of chronic disease increases with age. Understanding the relationships between the processes of aging and age-related diseases is an important initiative of the National Institutes of Health to improve the health of the aging population [1]. Slowing the aging process limits the burden of age-related chronic disease [2]. Identifying characteristics that slow aging may also provide approaches for preventing chronic diseases. Animals with increased lifespan aid in understanding the aging process by allowing the study of physiological and biochemical adaptations associated with slowed aging. Further, studying characteristics shared among long-lived models provides insight into pathways that are key to slowing the aging process and age-associated chronic diseases.

Lifespan can be extended by genetic, dietary, and pharmacological interventions. Additionally, multiple species have independently evolved long lifespan, including humans and naked mole rats, both of which live more than four times longer than predicted by body size [3]. Some of the earliest discoveries of lifespan extension were single-gene mutations associated with the insulin-like growth factor I (IGF-1) and growth hormone $(\mathrm{GH})$ pathways. These mice, including the Snell dwarf [4], are smaller than their heterozygote counterparts and significantly longer-lived, some by $40 \%$ or more compared with controls. Long-term caloric restriction is the most consistent dietary manipulation to extend lifespan and recent evidence suggests that short-term transient nutrition restriction prior to weaning, accomplished by litter enlargement, also increases mean and maximal lifespan in mice [5]. Pharmaceutical manipulation of lifespan is in its infancy, with evidence that rapamycin can extend lifespan in mice [6].

It is well established that oxidant stress increases with age across a variety of tissues, including cardiac [7] and skeletal muscle [8], liver [9], and brain [10], and is associated with a wide variety of chronic age-related diseases including cancer, neurodegeneration, sarcopenia, and cardiovascular disease. Although the oxidative stress theory of aging has received criticism [11], it remains true that oxidant stress is associated with the aging process. In response to oxidative stress, cells upregulate antioxidant pathways, including activation of the transcription factor nuclear factor (erythroid-derived 2)-like 
2 (Nrf2), the master regulator of antioxidant defenses and the proposed "master regulator" of the aging process [3]. Further, the therapeutic potential of Nrf2 is well supported in neurodegeneration and cancer (reviewed in $[12,13]$ ), highlighting a role for $\mathrm{Nrf} 2$ in attenuating age-related chronic disease. Below, we will review what is known about Nrf2 in four models of lifespan extension: caloric restriction, rapamycin feeding, short-term nutrition restriction, and the Snell dwarf mouse. Further, we will discuss what is known about Nrf2 in the exceptionally long-lived naked mole rat and in humans who show enhanced longevity, with the overall goal of describing Nrf2 signaling in longevity interventions and in naturally occurring models of long life.

\section{Nrf2 Signaling Basics}

A member of the basic leucine zipper transcription factor family, Nrf2, controls both basal and inducible expression of over 200 target genes. When cellular stress is low, Nrf2 is sequestered in the cytoplasm by its involvement in an inactive complex with the actin-binding protein Kelch-like ECH-associated protein 1 (Keap1). Under these conditions, Keap1 targets Nrf2 for ubiquitination and degradation by the $26 \mathrm{~S}$ proteasome system, resulting in basal low-level expression of Nrf2 [14]. However, when activated, Nrf2 translocates to the nucleus and transcriptionally upregulates its cytoprotective transcriptional program through binding to the antioxidant response element (ARE) in the promoter region of its target genes. Activation by reactive oxygen species (ROS) is the best understood mechanism of Nrf2 activation. Oxidant exposure modifies cysteine residues on Keap1 resulting in conformational changes that protect Nrf2 from targeting for ubiquitination and degradation [15], thus resulting in Nrf2 accumulation and activation. In addition to ROS and electrophilic species, Nrf2 can also be activated by phytochemicals [16-18], as well as various pharmaceuticals (reviewed in [19]) via overlapping and distinct mechanisms.

Nrf2 target genes exhibit antioxidant properties and facilitate cellular responses against xenobiotics. Antioxidant enzymes include $\mathrm{NAD}(\mathrm{P}) \mathrm{H}$ dehydrogenase quinone 1 (NQO1), heme oxygenase-1 (HO-1), peroxiredoxin 1 (Prdx1), superoxide dismutase-1 (SOD-1), and many enzymes involved in glutathione synthesis such as glutathione S-transferases (GSTs) and glutamate-cysteine ligase modifier (GCLM), the ratelimiting step in glutathione synthesis (reviewed in [20]). In addition, the cytoprotective properties of $\mathrm{Nrf} 2$ activation extend beyond these classic target genes, as other ARE-containing genes exhibit anti-inflammatory activity [21] and autophagic properties [22] and aid in proteasomal removal of oxidative damaged proteins [23]. Nrf2 also regulates its own expression. Two ARE-like motifs in the 5 flanking region of the Nrf2 promoter are responsible for the induction of Nrf2 upon activation [24], ensuring a feed-forward process with Nrf2 activation promoting its own expression and thus facilitating a profound cellular response to stress.

Studies of Nrf2 knockout animals highlight the importance of Nrf2 in cytoprotection. These animals display a diminished ability to activate prosurvival genes [30] and are more susceptible to a wide range of stressors including hyperoxia, lipopolysaccharide, cigarette smoke, and UV irradiation, as well as various chemical insults [31]. Nrf2 knockout animals display diminished basal activity of Nrf2regulated antioxidant enzymes [30], as well as inducible targets. In a transcriptional analysis of Nrf2 target genes, treatment with the Nrf2 activator 3H-1,2-dithiole-3-thione (D3T) induced 292 genes in wild type animals, compared to only 15 in Nrf2 knockout mice [32]. Together, investigations of Nrf2 knockout animals demonstrate diminished basal and inducible gene programs, as well as increased sensitivity to cell stresses, and support $\mathrm{Nrf} 2$ as an important transcriptional regulator of the cytoprotective program.

\section{Nrf2 and Aging}

The role of Nrf2 in responding to cytotoxic stressors is well defined. However, only within the last few years have studies elucidated how Nrf2 function changes with age and how changes in Nrf2 activity contribute to the aging phenotype. Aging is sufficient to diminish cardiac Nrf2-ARE binding activity [7], and aged Nrf2 knockout mice exhibit decreased expression of antioxidant target genes [8]. Disruptions in Nrf2-Keapl signaling have been reported in skeletal muscle from sedentary older humans [33] and cardiac muscle from aging rats [7]. Impaired liver Nrf2 transcriptional activity in old rats results in glutathione depletion and significant downregulation of Nrf2-regulated glutathione biosynthetic enzymes [34]. Aged mice show similar losses in cellular redox capacity to those observed in Nrf2 knockout mice $[34,35]$, suggesting that Nrf2 dysregulation with age may be responsible for the loss of cellular redox status. Diminished Nrf2 target gene expression with age is accompanied by increased muscle ROS production, glutathione depletion, and increased oxidant damage to proteins, DNA, and lipids in both humans [33] and rodents [34]. Therefore, given that Nrf2 activity decreases with age alongside increased oxidant stress, interventions that activate Nrf2 may impact the aging process and longevity.

Support for the role of Nrf2 in regulation of lifespan comes from $\mathrm{Nrf} 2$ gain of function and loss of function studies. For example, experimental deletion of the antielectrophilic gene glutathione transferase ( $g$ Gsta4) activated Nrf2 and significantly extended lifespan in mice [36]. This mutation increased electrophilic lipid peroxidation products and increased nuclear Nrf2 activity by $43 \%$ and $38 \%$ in liver and skeletal muscle, respectively. The authors propose that deletion of this glutathione transferase gene resulted in chronic moderate Nrf2 activation and presumably elevated downstream Nrf2 signaling throughout the mouse lifespan. Studies of the Nrf2 homolog SKN-1 in Caenorhabditis elegans (C. elegans) and the Drosophila homolog $\mathrm{CncC}$ further suggest that Nrf2 may be implicated in longevity processes. Upon activation, SKN-1 and $\mathrm{CncC}$ upregulate genes involved in the oxidative stress response, including many orthologs to those regulated by mammalian $\mathrm{Nrf2}[37,38]$. Similar to mouse Nrf2 knockouts, SKN-1 mutants show diminished resistance to oxidative stress and shortened lifespan. On the other hand, 
moderate overexpression of a constitutively active SKN-1 increases lifespan, alongside increased resistance to oxidative stress [37]. Similarly, Keap1 loss-of-function mutations extend the lifespan of male Drosophila [38]. Various lifespan extending genetic manipulations in C. elegans require SKN1. Dietary restriction activates $\mathrm{SKN}-1$, and expression of the transcription factor in C. elegans neuronal cells is required for longevity to be extended by dietary restriction [39]. The long-lived daf-2 (nematode homologue of FOXO) mutant increases lifespan in part through resultant activation of SKN-1 [40]. Together, what is known about Nrf2 and aging, alongside preliminary studies of the role of Nrf2 and SKN-1 in cytoprotection, suggests that loss of Nrf2 is important in ageassociated declines in oxidant stress resistance, and perhaps in the aging pathology itself.

\section{Nrf2 in Long-Lived Models}

4.1. Naked Mole Rat. The naked mole rat is an exceptionally long-lived species, with a lifespan four times longer than similarly sized rodents, thus making the naked mole rat an important model for longevity studies [41]. Naked mole rats do not have typical lifespan curves in which mortality rates increase with age, but rather they experience few of the biological changes typically associated with aging such as decreased metabolic rate, body composition changes, and declines in genomic and proteomic integrity [42]. Naked mole rats are resistant to age-associated diseases such as cancer [43], cardiac diastolic dysfunction [44], and neurodegenerative diseases [45]. Thus, the naked mole rat represents a naturally occurring, unique model of healthy aging.

Surprisingly, early studies of naked mole rats revealed that they have higher levels of oxidative damage, including lipid peroxidation products, protein carbonyls, and oxidative DNA modification in the liver, compared to shorter-lived mouse controls [46]. Subsequent comparisons of young and old naked mole rats revealed a striking difference between ageassociated changes in oxidative stress markers [47]. While macromolecular oxidant damage increases with age in mice, as it does in humans, naked mole rats maintain high levels of oxidative damage throughout their lifespan, similar to those observed in old mice [47]. In fact, few genes show differential expression between young and old animals, as assessed by transcriptome analyses in the brain, liver, and kidney from 4- and 20-month-old naked mole rats [26]. Therefore, while naked mole rats from a young age contain higher levels of oxidative damage than shorter-lived control mice, the typical age-associated increase in damage is blunted in this species, suggesting maintenance of oxidant stress defenses over time.

Naked mole rats also have significantly elevated proteasome quality control mechanisms [25]. The high breakdown and clearance of damaged proteins is suspected to be largely due to increased Nrf2 expression, as Nrf2 regulates the transcription of $\alpha$ and $\beta$ subunits of the 26S proteasome, as well as the selective autophagy cargo protein p62 [48-50]. In support of the hypothesized role of Nrf2 in naked mole rat longevity, under nonstressed conditions, naked mole rats have greater protein levels of Nrf2 including nuclear Nrf2, elevated Nrf2-ARE binding activity, and greater expression of Nrf2-regulated enzymes in fibroblasts and liver [51,52]. These data suggest $\mathrm{Nrf2}$ may be responsible for the heightened quality control mechanisms in naked mole rats and may be associated with their exceptional longevity.

4.2. Caloric Restriction. Caloric restriction (CR), a decrease in caloric intake without malnutrition, is the most consistent and robust means to increase lifespan across species, from flies to rodents [53], as well as nonhuman primate models [54]. Additionally, CR imparts slowed aging effects and delays the incidence of age-related disease [55]. Although the mechanisms underlying the effects of CR on longevity remain largely unknown, protection against carcinogenesis [56], reduced insulin/insulin-like growth factor (IGF) signaling [57], and prolonged survival [53] are documented. In addition, CR improves cellular adaptation to stress [58], as evidenced by liver mitochondria isolated from CR rats which show delayed opening of the mitochondrial transition pore upon oxidative challenge [58]. In an elegant study using sera collected from humans practicing long-term CR compared to sera collected from age- and sex-matched individuals following a typical western diet, treatment of cultured human primary fibroblasts with CR sera significantly upregulated gene expression of stress-response genes and enhanced tolerance to oxidants [59]. CR decreases ROS production, enhances the plasma membrane redox system, improves insulin signaling, and attenuates inflammation [27], all of which have been associated with improved age-related disease outcomes.

Many of the positive outcomes of CR have been associated with Nrf2 activation. For example, a variety of carcinogens activate Nrf2 and the ARE, protecting against carcinogenesis [60]. Changes in insulin levels, such as those elicited by fasting, elicit a small acute oxidant stress and subsequent activation of Nrf2 and its targets [61]. CR prevents the ageinduced loss of cellular antioxidant capacity, in part due to increased levels of Nrf2 targets NQO1 and GSTs in brain and liver [62,63]. In cerebral vascular endothelial cells, CR prevents the age-related decline in Nrf2 activity. Further, CR upregulates Nrf2 expression in old mice to a level that surpasses that of young ad libitum (AL) fed animals [64], highlighting the importance of Nrf2 with aging and its potential activation by longevity promoting interventions. Several proposed CR mimetics, such as resveratrol, quercetin, and curcumin [65], act to increase lifespan and slow aging at least in part through activation of Nrf2 [28, 66-68]. We assessed Nrf2 signaling in cardiac muscle from 7-month-old animals that underwent lifelong $40 \%$ reduction in caloric intake compared to mice fed AL. We found Nrf2 protein expression to be unchanged by CR. However, NQO1 and SOD-1 were significantly upregulated (Figure 1) in CR, suggesting that a lifelong reduction in caloric intake may activate Nrf2 and be implicated in longevity in these animals.

Despite the positive associations between CR and Nrf2, the seminal study of Nrf2 and CR-induced lifespan extension is conflicting [69]. At 20 weeks of age, male and female Nrf2 knockout and wild type mice initiated a $40 \%$ reduction in caloric intake. As anticipated, the wild type mice 

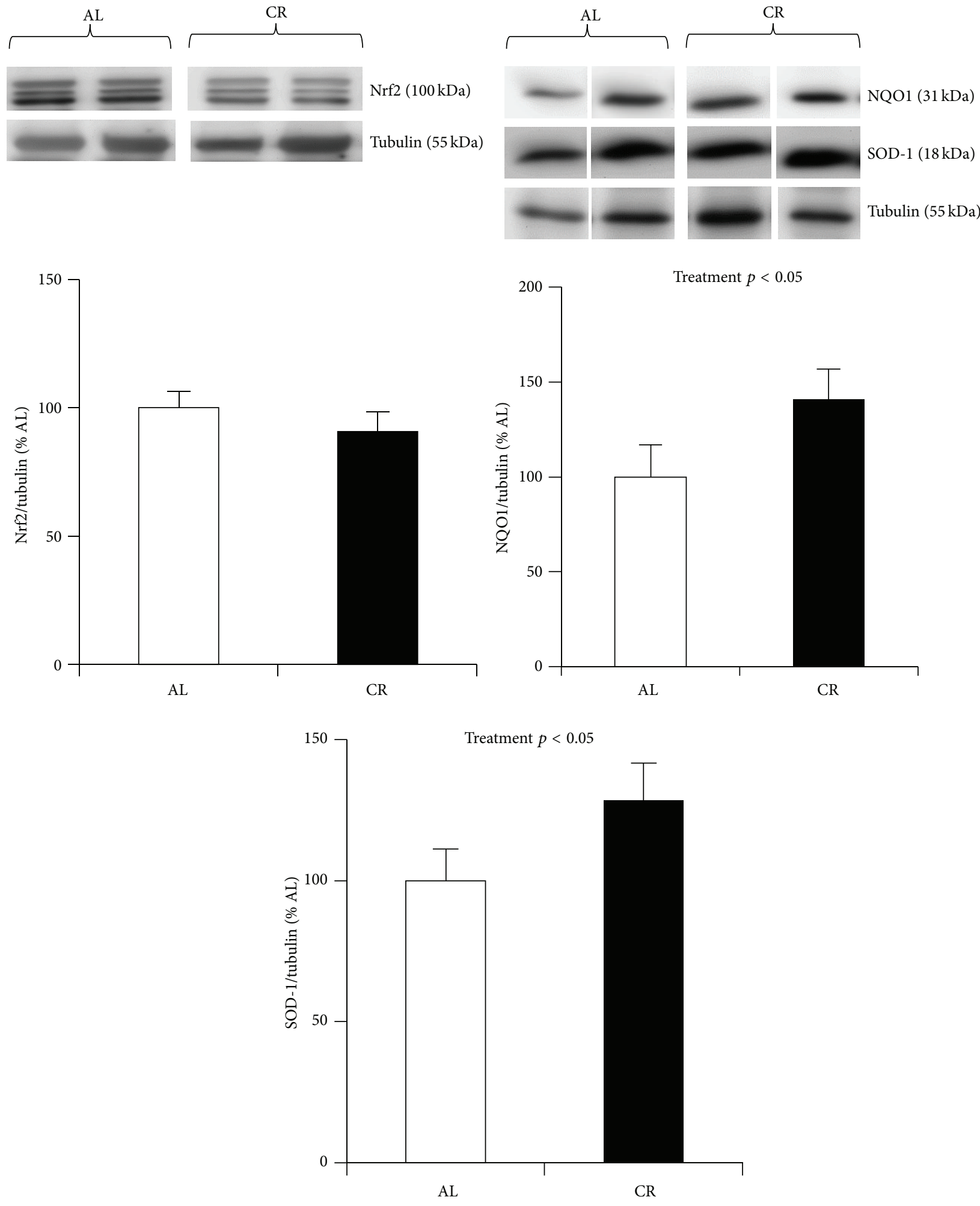

FIGURE 1: Nrf2 regulated protein expression in cardiac muscle from lifelong calorically restricted mice. Nrf2 was not different compared to ad libitum (AL) mice, while NQO1 and SOD-1 were significantly higher in cardiac muscle from CR mice compared to AL. Nrf2, NQO1, and SOD-1 were analyzed by western blotting and normalized to tubulin, shown below the proteins from each blot. Data are expressed as a ratio of target protein to tubulin (mean $\pm \mathrm{SEM}$ ). $n=6$ males in each condition. Lifelong B6D2F1 CR mice were maintained at the NIA colony at $40 \%$ food restriction compared with AL. Male mice were purchased at 6 months of age and divided into AL and CR groups. CR animals were maintained on NIH-31/NIA Fortified Diet, whereas AL animals were maintained on NIH-31 diet. 
were significantly protected against carcinogenesis on the CR diet compared to Nrf2 KO animals. However, survival curves showed that the CR and AL Nrf2 knockout mice overlapped until week 80 , when they began to deviate for the remainder of the lifespan study, with CR Nrf2 knockout mice significantly outliving AL Nrf2 knockout mice. Therefore, while CR protected against carcinogenesis in a manner that was dependent on Nrf2, genetic ablation of Nrf2 did not attenuate lifespan extension by CR. This study suggests that lifespan extension in mice by CR is not entirely dependent on upregulation of the Nrf2 pathway, despite its promising role as a chemoprevention target. However, this conclusion is not without criticism, and a role of the antioxidant response in CR-mediated longevity cannot be completely ruled out [29]. Future investigations into whether or not other agerelated diseases are slowed or reversed by Nrf2 activation and whether the attenuation of these diseases by $\mathrm{CR}$ requires $\mathrm{Nrf} 2$ are warranted.

4.3. Rapamycin. Rapamycin, a well-defined inhibitor of the mechanistic target of rapamycin (mTOR), interacts with the Nrf2 signaling pathway, and Nrf2 may be implicated in rapamycin-mediated longevity. Exposure of adult C. elegans to rapamycin activates SKN-1 and downstream gene targets and significantly increases oxidative stress resistance in SKN1 dependent manner [70]. Further, rapamycin treatment increased nematode lifespan through SKN-1, as evidenced by an abolished effect of rapamycin-mediated lifespan extension when SKN-1 was silenced by RNA interference [70]. Treatment with rapamycin also extends lifespan $[71,72]$ and slows the aging phenotype in mice, including decreased liver degeneration, attenuated cataract severity, and blunting of the age-associated decline in physical activity [73]. In vitro, chronic treatment with rapamycin significantly upregulates Nrf2 protein and transcript expression Nrf2 in fibroblasts, protects cultures from exogenous ROS exposure, and increases replicative lifespan of fibroblasts in culture [74]. We recently assessed skeletal muscle Nrf2 expression, as well as the expression of Nrf2 target proteins NQO1, HO-1, Prdxl, and SOD-1 in mice treated with rapamycin for 12 weeks. We were surprised to find no differences in Nrf2 and Nrf2 target protein expression between the long-lived rapamycin fed mice and controls. Prdxl expression in skeletal muscle was significantly lower than control, but no other differences were observed in Nrf2 target protein expression between longlived and control animals (Figure 2). Interestingly, we further assessed sex differences and found NQO1, Nrf2, and SOD-1 to be significantly greater in skeletal muscle from male mice compared to females (Figure 2). Given the disparate lifespan between males and females, and dissimilar effects of longevity interventions on male versus female mice, it is important to understand sex-specific signaling of the Nrf2 cytoprotective pathway, as elucidation of Nrf2 signaling in long-lived males and females may provide insight into the mechanisms behind sexual dimorphic longevity.

4.4. Snell Dwarf Mice. Snell dwarf mice are homozygous for a single-gene mutation at the Pit locus. This mutation results in an underdeveloped anterior pituitary and decreases in growth hormone $(\mathrm{GH})$ and insulin-like growth factor-1 (IGF1) signaling. These mice display a $40 \%$ increase in mean and maximal longevity in both male and female mice compared to mice on similar backgrounds [4] and show delay in many agerelated pathologies including attenuation of age-dependent collagen cross-linking and age-sensitive indices of immune system status [4]. This single gene mutation thus controls both maximal lifespan and the timing of senescence and agerelated pathology, supporting the role of the IGF-1 and GH pathways in regulating mammalian longevity.

Snell mice display heightened Nrf2 signaling, as evidenced by increased skin-derived fibroblast expression of total cell Nrf2 protein compared to controls, in addition to upregulated expression of Nrf2 targets HO-1, thioredoxin, and GCLM [75]. In addition, Snell-derived fibroblasts show enhanced resistance to various forms of cytotoxic stress, like paraquat, peroxide, cadmium, and others that kill cells in part via ROS [76]. We recently assessed Nrf2 and targets in skeletal and cardiac muscle from 7-month-old Snell mice and found no differences in Nrf2 and target protein expression compared to control mice (Figure 3) in either males or females. Part of the discordance in Nrf2 signaling in primary fibroblasts compared to skeletal and cardiac muscle may be due to tissue-specific differences. Previous work suggests that Nrf2 activators induce the Nrf2 transcriptional program in some cell types, but not others $[19,77]$, demonstrating that cell and tissue types respond quite differently to Nrf2 activators. Varying metabolic demands between tissues may be responsible for tissue-specific Nrf2 activity [77], or inherent properties of the tissue, such as expression of Nrf2 activators/inhibitors. An assessment of tissue-specific differences in cellular stress responses is lacking and may play an important role in determining the response of Nrf2 to different longevity interventions.

4.5. Crowded Litter. Recent evidence shows that very-shortterm nutrient restriction limited only to the preweaning phase by litter crowding extends lifespan by $16 \%$ in female mice and 7\% in males [5]. In this model termed crowded litter (CL), increasing litter size from eight pups per mother to 12 imposes a transient energy restriction thought to represent an epigenetic means of improving lifespan [78], as the energy stress imposed only in the first three weeks of life extends lifespan and healthspan [79]. At weaning and through adult life, CL mice are leaner and consume more oxygen relative to body mass compared to control mice and have improved lifelong alterations in metabolic status [79]. CL mice have less body fat, lower leptin levels, and higher glucose tolerance and are more insulin sensitive than control mice [79]. Despite few studies interrogating mechanisms by which CL intervention imparts improved healthspan and lifespan, it seems clear that long-lasting endocrine and metabolic effects result from the early-life nutrient restriction.

As studies of CL mice are in their infancy, minimal data thus far directly link Nrf2 and CL. However, investigations of xenobiotic metabolism and resistance to cytotoxic insult suggest that CL mice maintain elevated levels of xenobiotic 

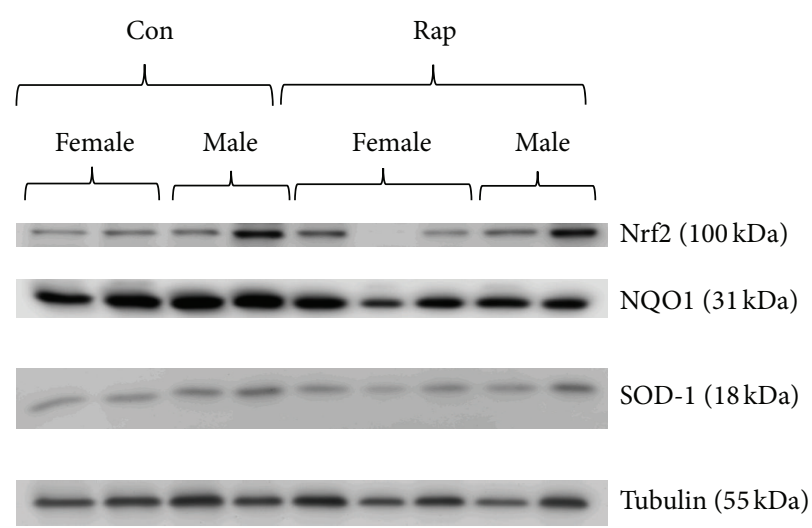

$\operatorname{Sex} p<0.05$

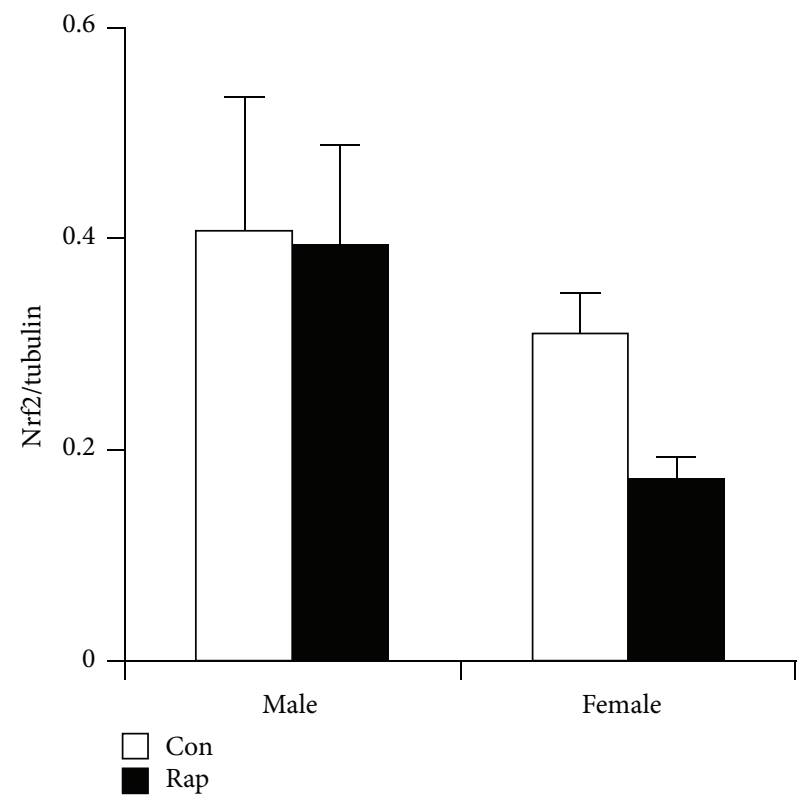

$\operatorname{Sex} p<0.05$

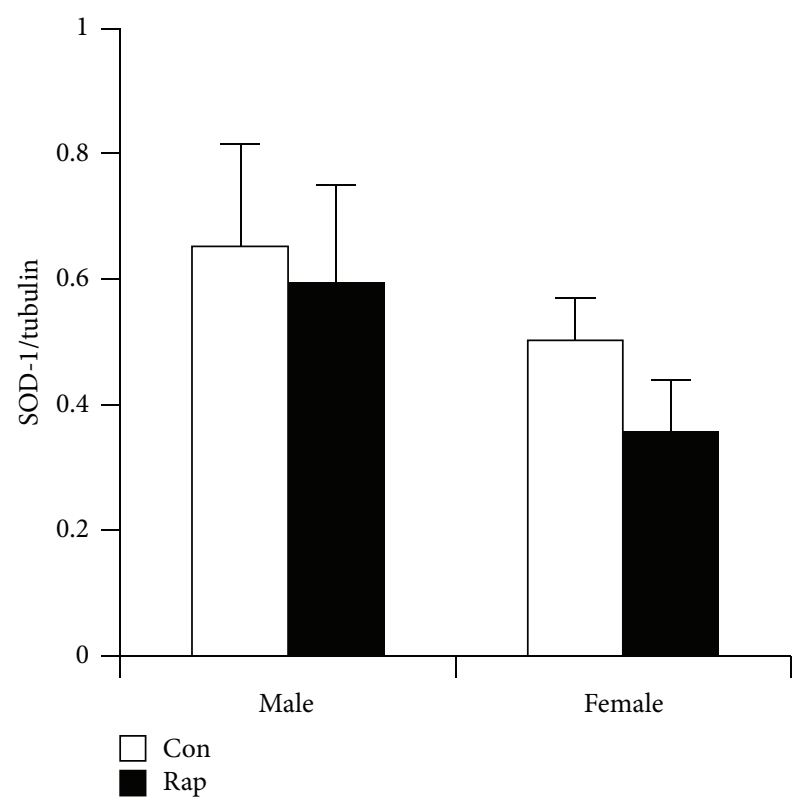

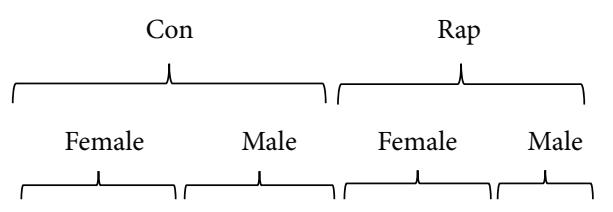

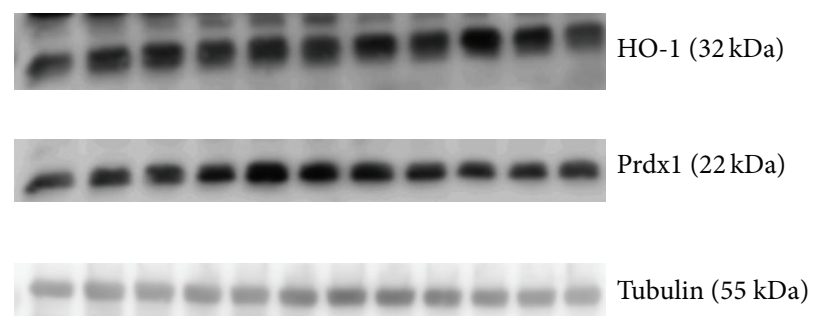

$\operatorname{Sex} p<0.05$
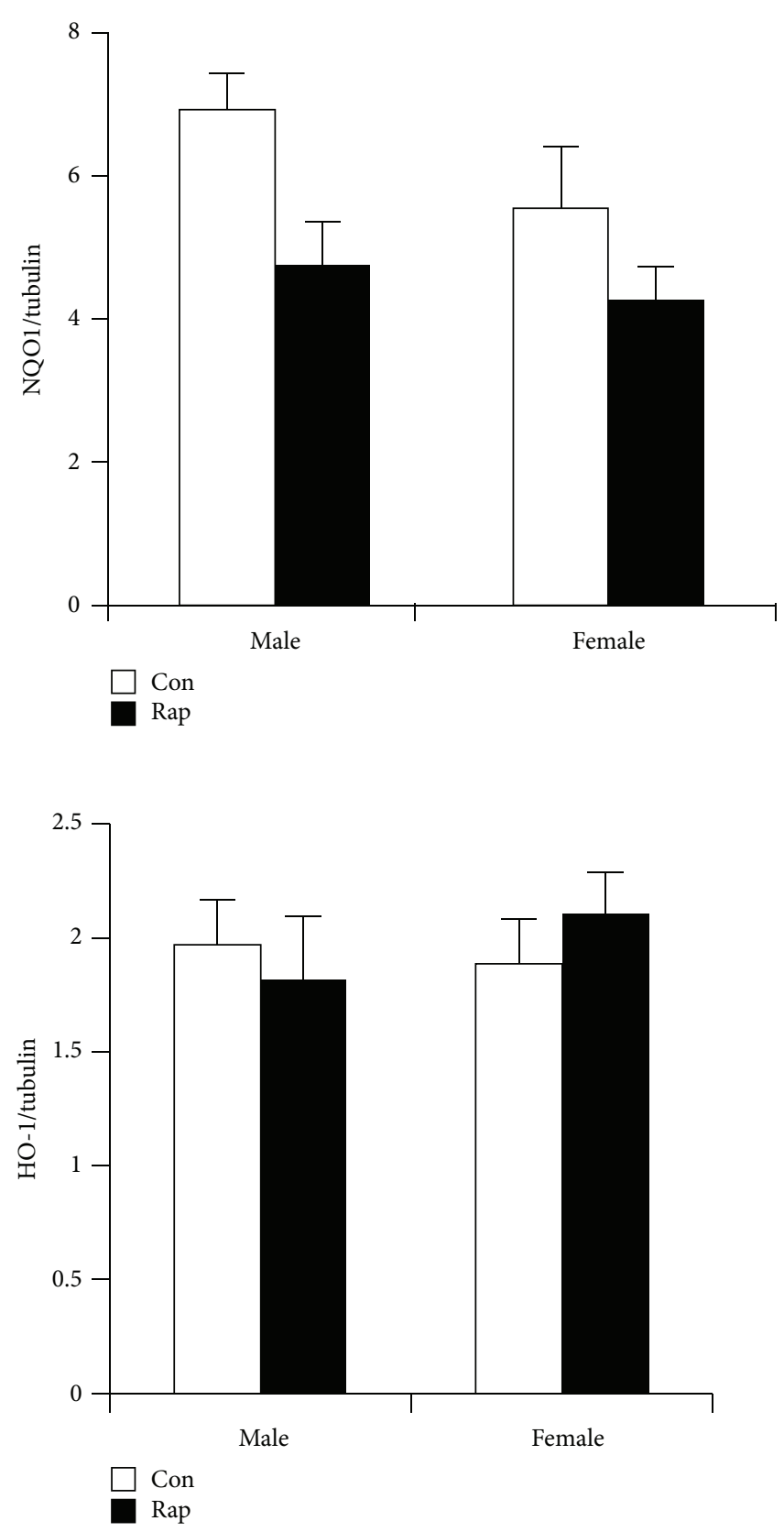

FIGURE 2: Continued. 


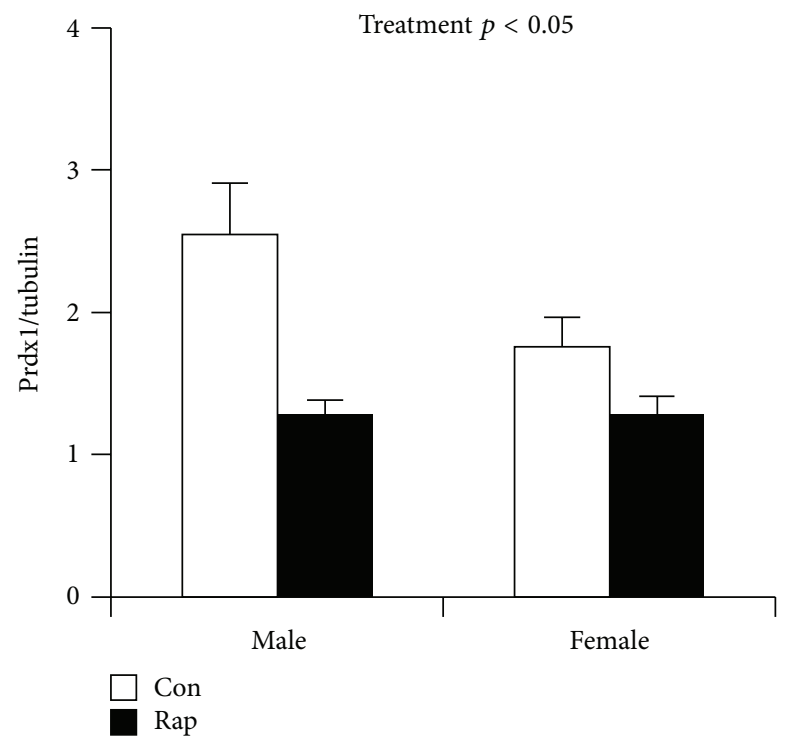

FIgURE 2: Nrf2 regulated protein expression in skeletal muscle from mice chronically fed with rapamycin. Nrf2, NQO1, and SOD-1 were significantly greater in skeletal muscle from male rapamycin (Rap.) treated mice. Chronic rapamycin feeding suppressed Prdxl expression compared to controls. Nrf2, NQO1, SOD-1, and Prdxl were analyzed by western blotting and normalized to tubulin, shown below the proteins from each blot. Data are expressed as a ratio of target protein to tubulin (mean $\pm \mathrm{SEM}$ ). $n=6$ males and $n=6$ females in each condition. UM-HET3 mice were generated by the offspring of crosses between (BALB/cByJ x C57BL/6J) F1 females and (C3H/HeJ x DBA/2J) F1 males. Mice were fed with chow mixed with encapsulated rapamycin at $14 \mathrm{mg} / \mathrm{kg}$ food (equivalent to $2.24 \mathrm{mg}$ of rapamycin/ $\mathrm{kg}$ body weight/day) or normal chow for 12 weeks in accordance with the original study describing lifespan extension [6].

phase I metabolizing enzymes in the liver, alongside heightened resistance against tert-butylhydroquinone [78], an oxidant known to activate Nrf2 [80]. Based on these data showing enhanced stress resistance in CL, we assessed Nrf2 and targets in skeletal muscle from 3-4-month-old CL mice. In this young cohort, we failed to find differences in Nrf2 targets between CL and control (Figure 4) in either male or female mice. As metabolic and hormonal status change throughout development in the CL mice, corresponding alterations in Nrf2 activation may occur. Given the early stages of this longevity intervention, few investigations have been conducted into developmental changes in CL mice and how metabolism and Nrf2 may interface to regulate longevity in this model. Future investigations are warranted to more completely assess whether Nrf2 mediates lifespan extension and what role Nrf2 may play throughout the lifespan of CL mice.

4.6. Humans. Humans are amongst the longest-lived mammals, with a maximum species lifespan potential (MLSP) of over 100 years and a predicted lifespan four times longer than estimated by body mass [42]. Exceptionally long-lived humans, centenarians, may have constitutively upregulated Nrf2, allowing them to better respond to cell stresses and minimize cell damage with age [81]. Investigations of centenarians who seem to reside in areas with high nutrient density and low caloric density diets support this hypothesis, as these diets may be characterized as a prolonged mild form of CR. Physical activity is one of the most effective interventions to prevent chronic disease and delay the detrimental cellular changes with age (reviewed in [82]). Acute exercise stress activates Nrf2 [83] and is associated with enhanced antioxidant capacity. Therefore, it is plausible that exercise, a longevity-promoting intervention in humans, may promote healthspan in part through activation of Nrf2. Nrf2 activators are currently undergoing phase II clinical trials for treatment of various human chronic diseases. Bardoxolone methyl therapy, a therapeutic pulmonary hypertension intervention [84], and dimethyl fumarate (BG-12), a currently approved therapy for relapsing-remitting multiple sclerosis, promote cytoprotective properties, in part through activation of Nrf2 [85]. A sulforaphane-based pharmaceutical is slated to begin trials for prostate cancer [86] following preliminary studies showing diminished Nrf2 signaling in prostate cancer that is restored by sulforaphane treatment [87]. Thus it appears that some of the human diseases associated with aging may be treated with Nrf2 activators. However, whether Nrf2 expression is higher in long-lived centenarians remains unknown. Further, whether Nrf2 activation can increase human lifespan or delay human aging is still unexplored.

4.7. Nrf2 Activity and Maximal Lifespan Potential. The simultaneous study of species with varying MLSP facilitates the identification of associations between species longevity and specific mediators of lifespan, such as Nrf2. A comparison of eight rodent species with widely divergent longevities ranging from 4 to 31 years yielded a positive association between Nrf2ARE binding activity and MLSP [52]. Quantitatively, for a 10-year increase in lifespan, Nrf2 activity increased 1.4-fold. Surprisingly, the authors of this study found that Nrf2 protein 

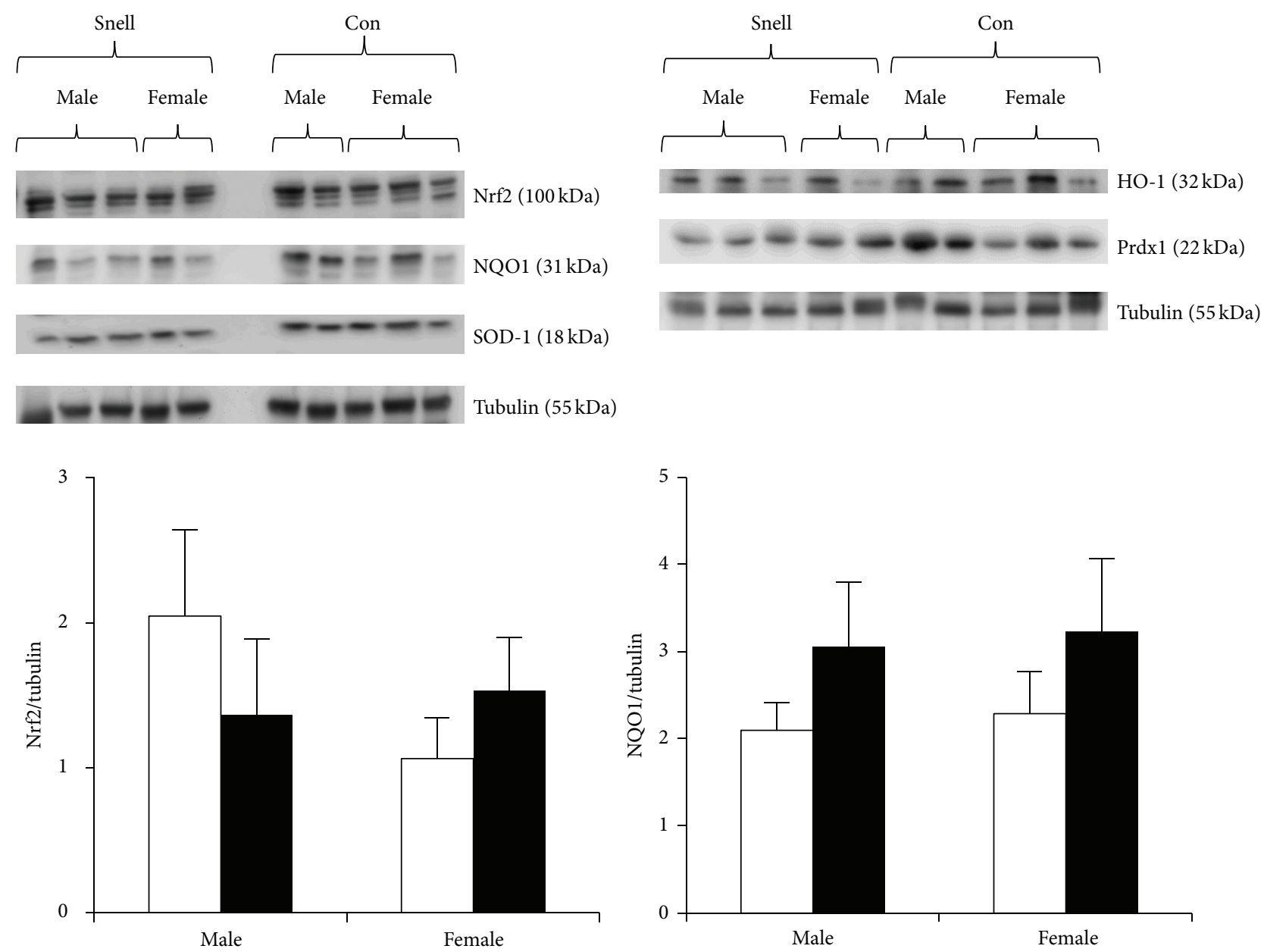

$\square$ Con
$\square$ Snell

$\square$ Con

- Snell
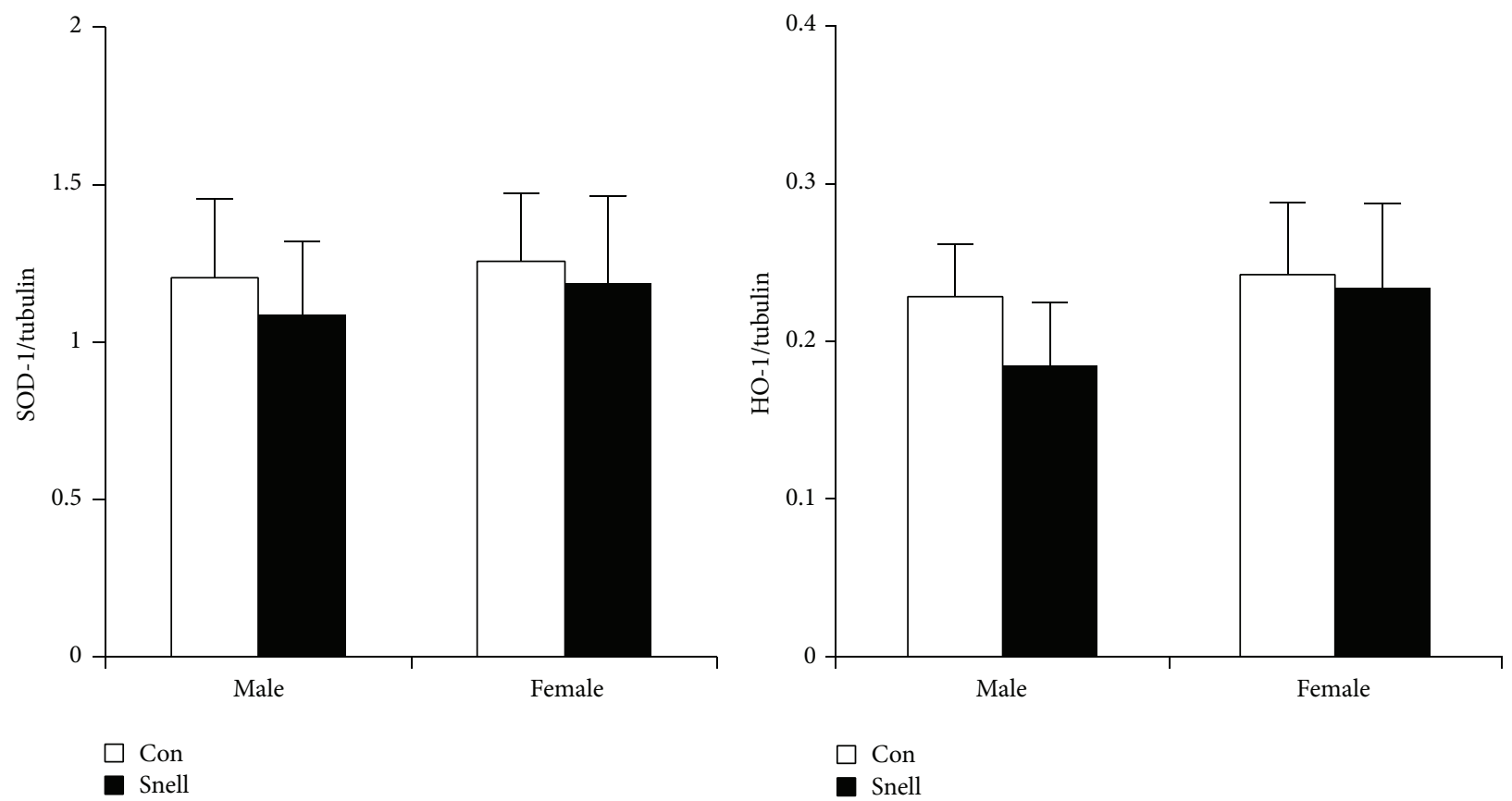

$\square$ Con

- Snell

FIGURE 3: Continued. 


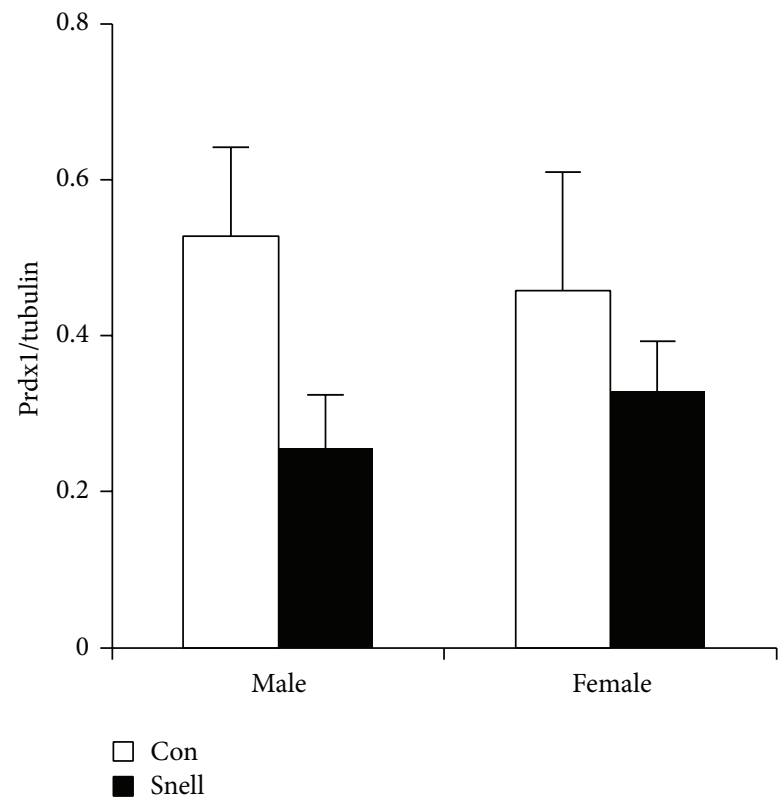

Figure 3: Nrf2 regulated protein expression in skeletal muscle from Snell dwarf mice. The Pit1 mutation does not influence Nrf2 or Nrf2 target protein expression in skeletal muscle. Nrf2, NQO1, HO-1, SOD-1, and Prdx1 were analyzed by western blotting and normalized to tubulin, shown below the proteins from each blot. Densitometric analyses were conducted on the multiple band of Nrf2. Data are expressed as a ratio of target protein to tubulin (mean \pm SEM). $n=10$ males and 10 females of each genotype. Snell dwarf $(d w / d w)$ and heterozygote $(d w /+)$ control mice were bred as the progeny of (DW/J x C3H/HeJ) F1 $d w /+$ females and (DW/J x C3H/HeJ) F1 $d w / d w$ males [4].

levels did not correlate with MLSP. Rather, mechanisms that control Nrf2 activity, such as Keap1 expression, significantly correlated with MLSP. Therefore, the authors posited that long-lived species are poised to effectively respond to cellular stresses associated with age and chronic disease due to the natural variation in $\mathrm{Nrf} 2$ activity. Together, these data support the notion that Nrf2 may be the "guardian of healthspan and the gatekeeper of species longevity" [3] and suggest that future investigations should elucidate whether activation of Nrf2 facilitates increases in healthspan and lifespan.

\section{Mechanisms of Nrf2 Activation: Basal versus Inducible Activity}

Our results of Nrf2 in long-lived mice presented here were obtained from young (3-4 and 7 months of age) mice that were not exposed to acute or chronic stresses. Young, unstressed animals generally do not display elevated Nrf2 signaling, as supported by investigations of young Nrf2 knockout mice, which do not have evidence of redox imbalance as assessed by either expression of Nrf2 target genes, overall cellular antioxidant status, or overt macromolecule oxidant damage [83]. However, when these Nrf2 knockout mice undergo an acute stress or when mice age, the ability to respond to and recover from the stress is attenuated. Thus, we suggest that the young mice in the long-lived cohorts reviewed here did not display enhanced Nrf2 signaling because there was no stressful stimulus from which to necessitate enhanced Nrf2 activation. Further, we assessed Nrf2 protein expression, as well as downstream
Nrf2 target expression. Previous correlations between Nrf2 protein expression and MSLP, however, show no association between these two measurements [52]. Instead, Nrf2 activity, as assessed by Nrf2-ARE binding activity, along with proteins that influence Nrf2 activity, such as Keap1 expression [38], predicted MLSP. Therefore, we suggest that future investigations of Nrf2-mediated longevity should assess Nrf2 activity and the key proteins that regulate Nrf2 activation and nuclear localization. Further, we propose that these investigations be conducted in aged animals or younger animals administered a stressful stimulus. It is under these conditions that differences in Nrf2 signaling between long-lived and control animals should be most apparent.

\section{Conclusions and Future Directions}

Chronic disease incidence increases with age. Slowing the aging process limits the burden of chronic disease [2]. The transcription factor Nrf2, a proposed "master regulator of the aging process," regulates a wide battery of cytoprotective responses and helps attenuate age-related disease, and its activity is positively associated with species lifespan potential. Previous work of Nrf2 in long-lived models shows promise for slowed aging interventions (summarized in Table 1), with naked mole rats, an exceptionally long-lived species, showing enhanced Nrf2 signaling compared to shorter-lived mouse species. Caloric restriction and many of its pharmaceutical mimetics activate $\mathrm{Nrf} 2$ and protect against age-associated carcinogenesis, despite contradicting evidence showing no effect of Nrf2 expression in CR-induced longevity. Studies 


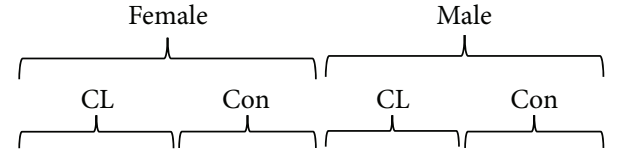

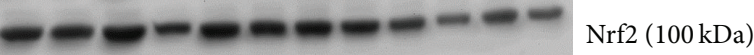

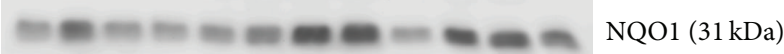

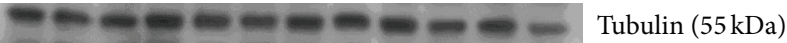

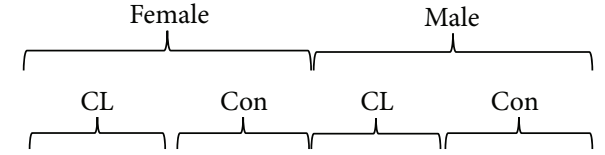

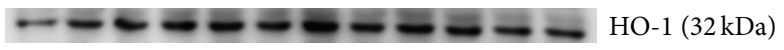
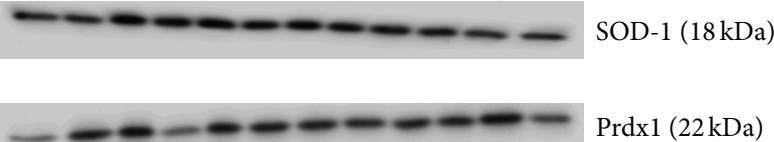

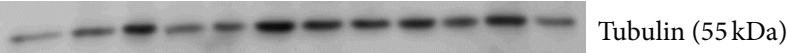

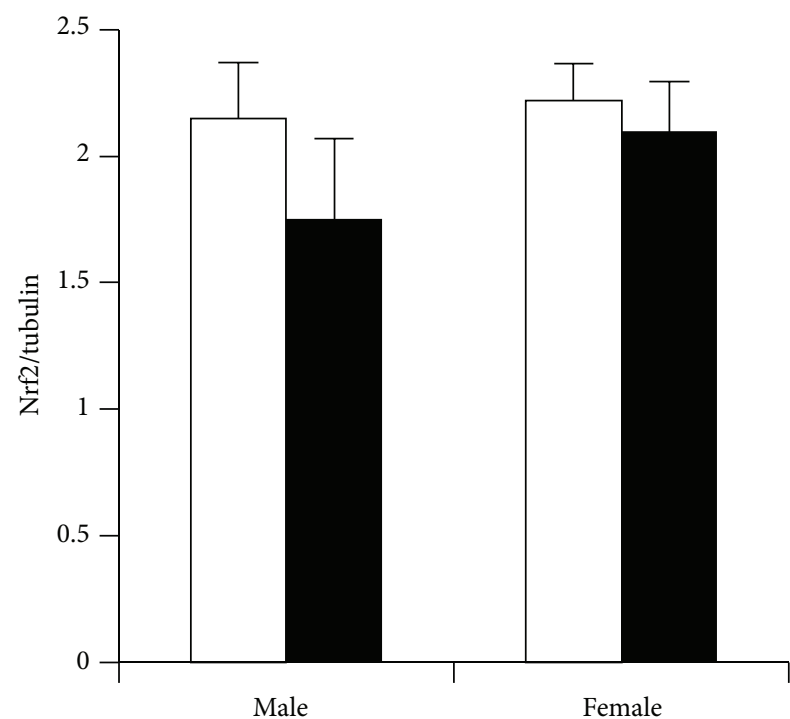

$\square$ Con

$\square \mathrm{CL}$

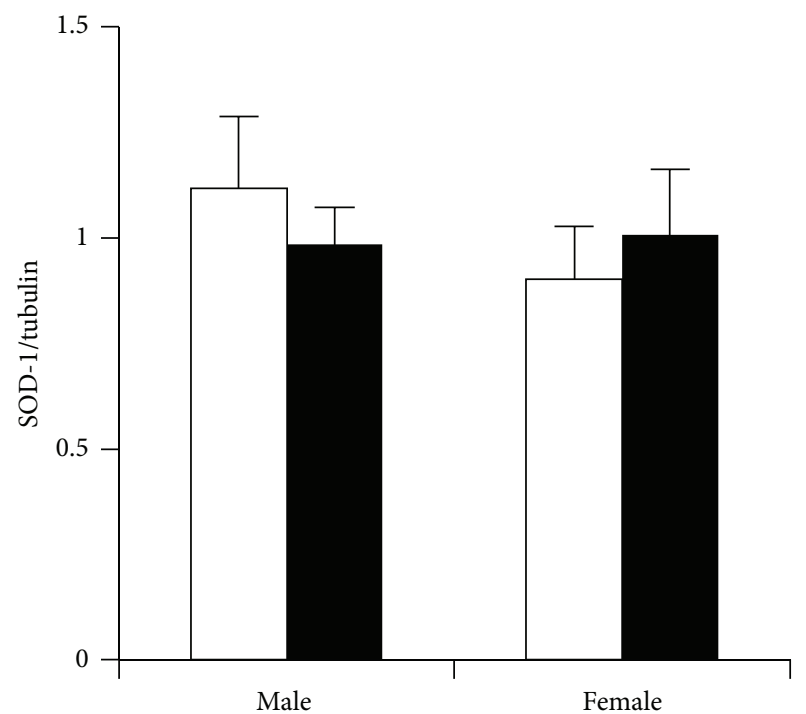

$\square$ Con

$\square \mathrm{CL}$

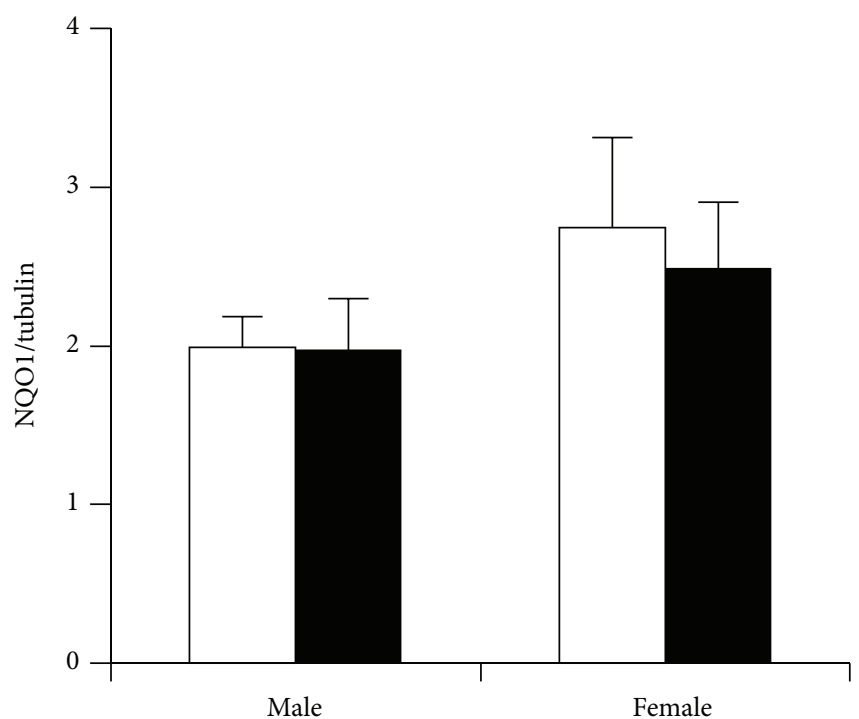

$\square$ Con

- $\mathrm{CL}$

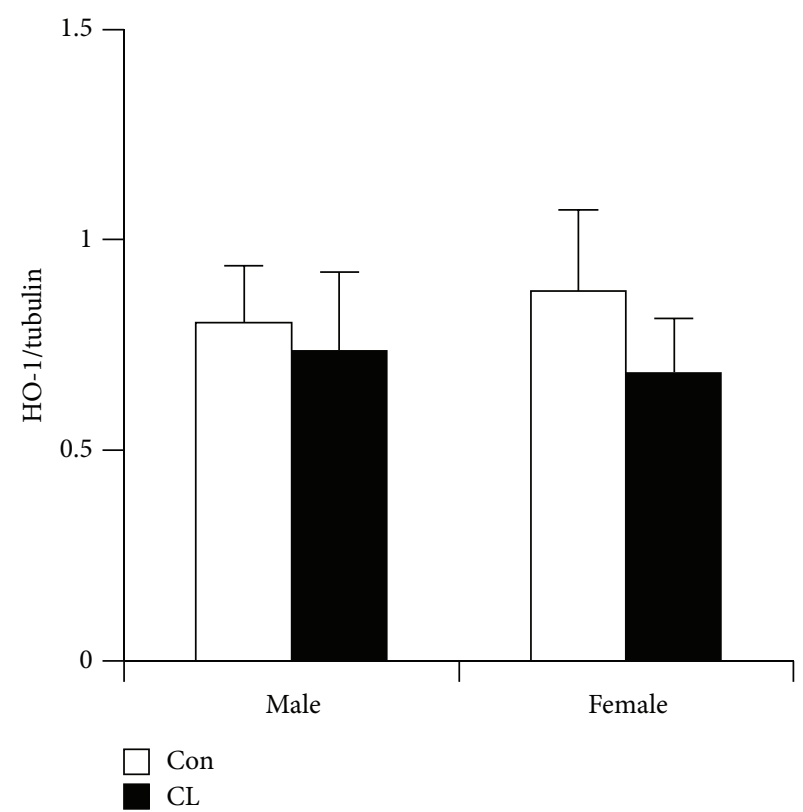

FIgURE 4: Continued. 


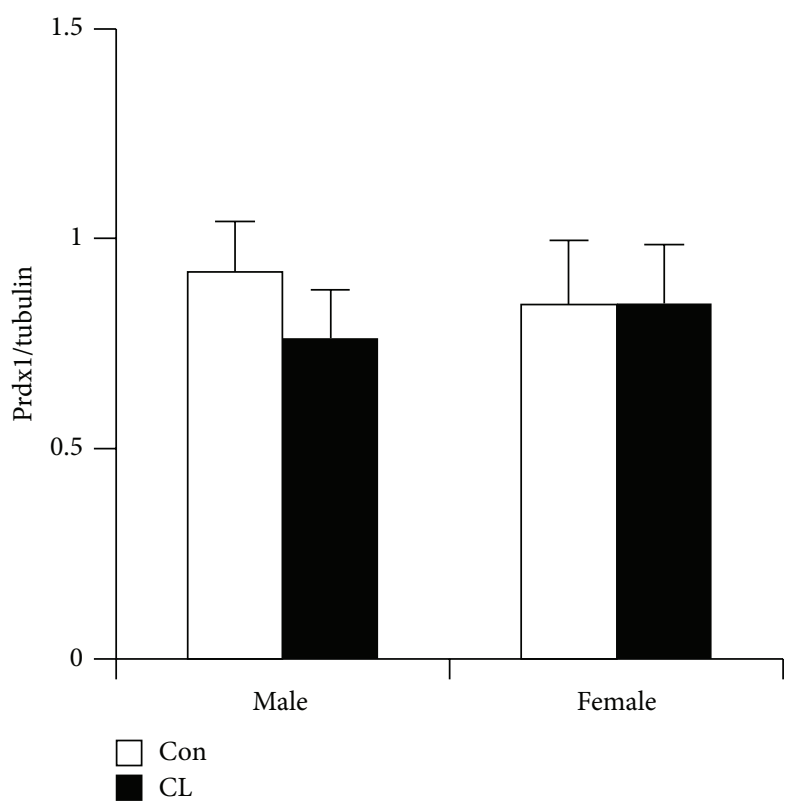

FIGURE 4: Nrf2 regulated protein expression in skeletal muscle from crowded litter mice. No significant sex or model differences were observed in skeletal muscle from crowded litter (CL) animals. Nrf2, NQO1, SOD-1, and Prdx1 were analyzed by western blotting and normalized to tubulin, shown below the proteins from each blot. Data are expressed as a ratio of target protein to tubulin (mean \pm SEM). $n=8$ males and $n=8$ females in each condition. CL mice, 3-4 months old, were generated following the previously described procedure [5]. UM-HET3 litters were culled to eight pups (control) or supplemented by transfer of newborn mice to produce litters of 12 mice (CL). Litters were weaned at three weeks of age, after which both groups were fed ad libitum.

TABLE 1: Summary of Nrf2 expression, Nrf2 target expression, and Nrf2 activity in long-lived models.

\begin{tabular}{|c|c|c|c|c|c|}
\hline Model & Tissue/cell type & Nrf2 expression & $\begin{array}{c}\text { Nrf2 target expression } \\
\text { (NQO1, HO-1, SOD-1, etc.) }\end{array}$ & $\begin{array}{c}\text { Nrf2 activity } \\
\text { (Nrf2-ARE binding) }\end{array}$ & Reference \\
\hline \multirow{2}{*}{ Naked mole rat } & Liver & $\uparrow$ & $\uparrow$ & $\uparrow$ & {$[25]$} \\
\hline & Fibroblast & $\uparrow$ & $\uparrow$ & $\uparrow$ & [26] \\
\hline \multirow{2}{*}{ Caloric restriction } & Heart & $\leftrightarrow$ & $\uparrow$ & N.R. & This manuscript \\
\hline & CMVEC & $\uparrow$ & N.R. & $\uparrow$ & [27] \\
\hline \multirow{2}{*}{ Rapamycin } & SkM & $\leftrightarrow$ & $\leftrightarrow$ & N.R. & This manuscript \\
\hline & C. elegans & $\uparrow$ & $\uparrow$ & $\uparrow$ & {$[28]$} \\
\hline \multirow{3}{*}{ Snell } & SkM & $\leftrightarrow$ & $\leftrightarrow$ & N.R. & This manuscript \\
\hline & Heart & $\leftrightarrow$ & $\leftrightarrow$ & N.R. & This manuscript \\
\hline & Fibroblast & $\uparrow$ & $\uparrow$ & N.R. & [29] \\
\hline Crowded litter & SkM & $\leftrightarrow$ & $\leftrightarrow$ & N.R. & This manuscript \\
\hline Humans & - & - & - & - & \\
\hline
\end{tabular}

Arrows indicate difference in the long-lived model compared to corresponding control, with $\uparrow$ indicating "greater in the long-lived model compared to control," $\leftrightarrow$ indicating "no difference between long-lived model and control," and N.R. indicating "data were not reported in the investigation." To date, no experimental data for Nrf2 expression or activity have been reported in aged humans. CMVEC: cerebromicrovascular endothelial cells; SkM: skeletal muscle (mixed skeletal muscle: gastrocnemius, soleus, and plantaris).

of rapamycin treated, Snell dwarf, and CL mice suggest that primary cells from these animals are more resistant to cytotoxic stress, which may be linked to elevated Nrf2 signaling. Future investigations should identify whether aged animals from long-lived cohorts have enhanced Nrf2 signaling that may explain their stress resistance. We hypothesize that pharmaceutical, genetic, epigenetic, and dietary manipulations that extend lifespan will enhance Nrf2 activation at advanced ages and under stressful cellular conditions, contributing to stress resistance and extended healthspan. Further, future investigations of Nrf2 signaling in humans, and the ability of Nrf2 activation to prevent chronic disease associated with aging, will lend further insight into the role of $\mathrm{Nrf} 2$ activation as a possible longevity-promoting intervention. 


\section{Abbreviations}

Nrf2: NFE2L2, nuclear factor (erythroid-derived 2)-like 2

SOD-1: Superoxide dismutase-1

NQO1: NAD $(\mathrm{P}) \mathrm{H}$ hydroquinone oxidoreductase-1

HO-1: Heme oxygenase-1

Prdxl: Peroxiredoxin 1

CL: $\quad$ Crowded litter

CR: Caloric restriction

Rap: Rapamycin

MLSP: Maximum species lifespan potential.

\section{Disclosure}

Benjamin F. Miller and Karyn L. Hamilton are co-principal investigators.

\section{Conflict of Interests}

The authors declare that there is no conflict of interests regarding the publication of this paper.

\section{References}

[1] J. B. Burch, A. D. Augustine, L. A. Frieden et al., "Advances in geroscience: impact on healthspan and chronic disease," The Journals of Gerontology, Series A: Biological Sciences and Medical Sciences, vol. 69, supplement 1, pp. S1-S3, 2014.

[2] A. Galioto, L. J. Dominguez, A. Pineo et al., "Cardiovascular risk factors in centenarians," Experimental Gerontology, vol. 43, no. 2, pp. 106-113, 2008.

[3] K. N. Lewis, J. Mele, J. D. Hayes, and R. Buffenstein, "Nrf2, a guardian of healthspan and gatekeeper of species longevity," Integrative and Comparative Biology, vol. 50, no. 5, pp. 829-843, 2010.

[4] K. Flurkey, J. Papaconstantinou, R. A. Miller, and D. E. Harrison, "Lifespan extension and delayed immune and collagen aging in mutant mice with defects in growth hormone production," Proceedings of the National Academy of Sciences of the United States of America, vol. 98, no. 12, pp. 6736-6741, 2001.

[5] L. Sun, A. A. S. Akha, R. A. Miller, and J. M. Harper, "Lifespan extension in mice by preweaning food restriction and by methionine restriction in middle age," Journals of Gerontology, Series A: Biological Sciences and Medical Sciences, vol. 64, no. 7, pp. 711-722, 2009.

[6] D. E. Harrison, R. Strong, Z. D. Sharp et al., "Rapamycin fed late in life extends lifespan in genetically heterogeneous mice," Nature, vol. 460, no. 7253, pp. 392-395, 2009.

[7] S. S. Gounder, S. Kannan, D. Devadoss et al., "Impaired transcriptional activity of Nrf2 in age-related myocardial oxidative stress is reversible by moderate exercise training," PLoS ONE, vol. 7, no. 9, Article ID e45697, 2012.

[8] C. J. Miller, S. S. Gounder, S. Kannan et al., "Disruption of Nrf2/ARE signaling impairs antioxidant mechanisms and promotes cell degradation pathways in aged skeletal muscle," Biochimica et Biophysica Acta, vol. 1822, no. 6, pp. 1038-1050, 2012.

[9] N. Sanz, C. Díez-Fernández, A. Alvarez, and M. Cascales, "Agedependent modifications in rat hepatocyte antioxidant defense systems," Journal of Hepatology, vol. 27, no. 3, pp. 525-534, 1997.
[10] G. Benzi, O. Pastoris, F. Marzatico, and R. F. Villa, "Age-related effect induced by oxidative stress on the cerebral glutathione system," Neurochemical Research, vol. 14, no. 5, pp. 473-481, 1989.

[11] S. I. Liochev, "Reflections on the theories of aging, of oxidative stress, and of science in general. is it time to abandon the free radical (oxidative stress) theory of aging?" Antioxidants \& Redox Signaling, 2014.

[12] M. J. Calkins, D. A. Johnson, J. A. Townsend et al., "The Nrf2/ARE pathway as a potential therapeutic target in neurodegenerative disease," Antioxidants and Redox Signaling, vol. 11, no. 3, pp. 497-508, 2009.

[13] C. L. L. Saw and A.-N. T. Kong, "Nuclear factor-erythroid 2-related factor 2 as a chemopreventive target in colorectal cancer," Expert Opinion on Therapeutic Targets, vol. 15, no. 3, pp. 281-295, 2011.

[14] K. Itoh, N. Wakabayashi, Y. Katoh et al., "Keap1 represses nuclear activation of antioxidant responsive elements by Nrf2 through binding to the amino-terminal Neh2 domain," Genes and Development, vol. 13, no. 1, pp. 76-86, 1999.

[15] K. I. Tong, A. Kobayashi, F. Katsuoka, and M. Yamamoto, “Twosite substrate recognition model for the Keapl-Nrf2 system: a hinge and latch mechanism," Biological Chemistry, vol. 387, no. 10-11, pp. 1311-1320, 2006.

[16] Y.-J. Surh, J. K. Kundu, and H.-K. Na, "Nrf2 as a master redox switch in turning on the cellular signaling involved in the induction of cytoprotective genes by some chemopreventive phytochemicals," Planta Medica, vol. 74, no. 13, pp. 1526-1539, 2008.

[17] E. L. Donovan, J. M. McCord, D. J. Reuland, B. F. Miller, and K. L. Hamilton, "Phytochemical activation of Nrf2 protects human coronary artery endothelial cells against an oxidative challenge," Oxidative Medicine and Cellular Longevity, vol. 2012, Article ID 132931, 9 pages, 2012.

[18] D. J. Reuland, S. Khademi, C. J. Castle et al., "Upregulation of phase II enzymes through phytochemical activation of Nrf2 protects cardiomyocytes against oxidant stress," Free Radical Biology and Medicine, vol. 56, pp. 102-111, 2013.

[19] B. M. Hybertson, B. Gao, S. K. Bose, and J. M. McCord, "Oxidative stress in health and disease: the therapeutic potential of Nrf2 activation," Molecular Aspects of Medicine, vol. 32, no. 46, pp. 234-246, 2011.

[20] M. Kobayashi and M. Yamamoto, "Nrf2-Keap1 regulation of cellular defense mechanisms against electrophiles and reactive oxygen species," Advances in Enzyme Regulation, vol. 46, no. 1, pp. 113-140, 2006.

[21] J. Kim, Y.-N. Cha, and Y.-J. Surh, "A protective role of nuclear factor-erythroid 2-related factor-2 (Nrf2) in inflammatory disorders," Mutation Research, vol. 690, no. 1-2, pp. 12-23, 2010.

[22] A. Jain, T. Lamark, E. Sjøttem et al., "p62/SQSTM1 is a target gene for transcription factor NRF2 and creates a positive feedback loop by inducing antioxidant response element-driven gene transcription," The Journal of Biological Chemistry, vol. 285, no. 29, pp. 22576-22591, 2010.

[23] A. M. Pickering, R. A. Linder, H. Zhang, H. J. Forman, and K. J. A. Davies, "Nrf2-dependent induction of proteasome and $\mathrm{Pa} 28 \alpha \beta$ regulator are required for adaptation to oxidative stress," Journal of Biological Chemistry, vol. 287, no. 13, pp. 1002110031, 2012.

[24] M.-K. Kwak, K. Itoh, M. Yamamoto, and T. W. Kensler, "Enhanced expression of the transcription factor Nrf2 by cancer 
chemopreventive agents: role of antioxidant response elementlike sequences in the nrf2 promoter," Molecular and Cellular Biology, vol. 22, no. 9, pp. 2883-2892, 2002.

[25] V. I. Pérez, R. Buffenstein, V. Masamsetti et al., "Protein stability and resistance to oxidative stress are determinants of longevity in the longest-living rodent, the naked mole-rat," Proceedings of the National Academy of Sciences of the United States of America, vol. 106, no. 9, pp. 3059-3064, 2009.

[26] E. B. Kim, X. Fang, A. A. Fushan et al., "Genome sequencing reveals insights into physiology and longevity of the naked mole rat," Nature, vol. 479, no. 7372, pp. 223-227, 2011.

[27] A. Martín-Montalvo, J. M. Villalba, P. Navas, and R. de Cabo, "NRF2, cancer and calorie restriction," Oncogene, vol. 30, no. 5, pp. 505-520, 2011.

[28] T.-C. Hsieh, X. Lu, Z. Wang, and J. M. Wu, "Induction of quinone reductase NQO1 by resveratrol in human K562 cells involves the antioxidant response element ARE and is accompanied by nuclear translocation of transcription factor Nrf2," Medicinal Chemistry, vol. 2, no. 3, pp. 275-285, 2006.

[29] G. P. Sykiotis and D. Bohmann, "Stress-activated cap'n'collar transcription factors in aging and human disease," Science Signaling, vol. 3, no. 112, p. re3, 2010.

[30] H. Zhu, K. Itoh, M. Yamamoto, J. L. Zweier, and Y. Li, "Role of Nrf2 signaling in regulation of antioxidants and phase 2 enzymes in cardiac fibroblasts: protection against reactive oxygen and nitrogen species-induced cell injury," FEBS Letters, vol. 579, no. 14, pp. 3029-3036, 2005.

[31] H. Motohashi and M. Yamamoto, "Nrf2-Keap1 defines a physiologically important stress response mechanism," Trends in Molecular Medicine, vol. 10, no. 11, pp. 549-557, 2004.

[32] M.-K. Kwak, N. Wakabayashi, K. Itoh, H. Motohashi, M. Yamamoto, and T. W. Kensler, "Modulation of gene expression by cancer chemopreventive dithiolethiones through the Keap1Nrf2 pathway. Identification of novel gene clusters for cell survival," Journal of Biological Chemistry, vol. 278, no. 10, pp. 8135-8145, 2003.

[33] A. Safdar, J. deBeer, and M. A. Tarnopolsky, "Dysfunctional Nrf2-Keap1 redox signaling in skeletal muscle of the sedentary old," Free Radical Biology and Medicine, vol. 49, no. 10, pp. 14871493, 2010.

[34] J. H. Suh, S. V. Shenvi, B. M. Dixon et al., "Decline in transcriptional activity of Nrf2 causes age-related loss of glutathione synthesis, which is reversible with lipoic acid," Proceedings of the National Academy of Sciences of the United States of America, vol. 101, no. 10, pp. 3381-3386, 2004.

[35] T. W. Kensler, N. Wakabayashi, and S. Biswal, "Cell survival responses to environmental stresses via the Keap1-Nrf2-ARE pathway," Annual Review of Pharmacology and Toxicology, vol. 47, pp. 89-116, 2007.

[36] S. P. Singh, M. Niemczyk, D. Saini, V. Sadovov, L. Zimniak, and P. Zimniak, "Disruption of the mgsta4 gene increases life span of C57BL mice," Journals of Gerontology-Series A: Biological Sciences and Medical Sciences, vol. 65, no. 1, pp. 14-23, 2010.

[37] J. H. An, K. Vranas, M. Lucke et al., "Regulation of the Caenorhabditis elegans oxidative stress defense protein SKN1 by glycogen synthase kinase-3," Proceedings of the National Academy of Sciences of the United States of America, vol. 102, no. 45, pp. 16275-16280, 2005.

[38] G. P. Sykiotis and D. Bohmann, "Keap1/Nrf2 signaling regulates oxidative stress tolerance and lifespan in Drosophila," Developmental Cell, vol. 14, no. 1, pp. 76-85, 2008.
[39] A. Abdullah, N. R. Kitteringham, R. E. Jenkins et al., "Analysis of the role of Nrf2 in the expression of liver proteins in mice using two-dimensional gel-based proteomics," Pharmacological Reports, vol. 64, no. 3, pp. 680-697, 2012.

[40] J. M. A. Tullet, M. Hertweck, J. H. An et al., "Direct inhibition of the longevity-promoting factor SKN-1 by insulin-like signaling in C. elegans," Cell, vol. 132, no. 6, pp. 1025-1038, 2008.

[41] R. Buffenstein, "Negligible senescence in the longest living rodent, the naked mole-rat: insights from a successfully aging species," Journal of Comparative Physiology B: Biochemical, Systemic, and Environmental Physiology, vol. 178, no. 4, pp. 439445, 2008.

[42] K. N. Lewis, B. Andziak, T. Yang, and R. Buffenstein, "The naked mole-rat response to oxidative stress: just deal with it," Antioxidants and Redox Signaling, vol. 19, no. 12, pp. 1388-1399, 2013.

[43] S. Liang, J. Mele, Y. Wu, R. Buffenstein, and P. J. Hornsby, "Resistance to experimental tumorigenesis in cells of a longlived mammal, the naked mole-rat (Heterocephalus glaber)," Aging Cell, vol. 9, no. 4, pp. 626-635, 2010.

[44] K. M. Grimes, M. L. Lindsey, J. A. L. Gelfond, and R. Buffenstein, "Getting to the heart of the matter: age-related changes in diastolic heart function in the longest-lived rodent, the naked mole rat," Journals of Gerontology-Series A: Biological Sciences and Medical Sciences, vol. 67, no. 4, pp. 384-394, 2012.

[45] Y. H. Edrey, D. Casper, D. Huchon et al., "Sustained high levels of neuregulin-1 in the longest-lived rodents; a key determinant of rodent longevity," Aging Cell, vol. 11, no. 2, pp. 213-222, 2012.

[46] B. Andziak, T. P. O’Connor, W. Qi et al., "High oxidative damage levels in the longest-living rodent, the naked mole-rat," Aging Cell, vol. 5, no. 6, pp. 463-471, 2006.

[47] B. Andziak and R. Buffenstein, "Disparate patterns of agerelated changes in lipid peroxidation in long-lived naked molerats and shorter-lived mice," Aging Cell, vol. 5, no. 6, pp. 525-532, 2006.

[48] G. Bjørkøy, T. Lamark, and T. Johansen, "p62/SQSTM1: a missing link between protein aggregates and the autophagy machinery," Autophagy, vol. 2, no. 2, pp. 138-139, 2006.

[49] E. N. Tsakiri, G. P. Sykiotis, I. S. Papassideri, V. G. Gorgoulis, D. Bohmann, and I. P. Trougakos, "Differential regulation of proteasome functionality in reproductive vs. somatic tissues of Drosophila during aging or oxidative stress," The FASEB Journal, vol. 27, no. 6, pp. 2407-2420, 2013.

[50] E. N. Tsakiri, G. P. Sykiotis, I. S. Papassideri et al., "Proteasome dysfunction in Drosophila signals to an Nrf2-dependent regulatory circuit aiming to restore proteostasis and prevent premature aging," Aging Cell, vol. 12, no. 5, pp. 802-813, 2013.

[51] K. N. Lewis, J. Mele, P. J. Hornsby, and R. Buffenstein, "Stress resistance in the naked mole-rat: the bare essentials-a minireview," Gerontology, vol. 58, no. 5, pp. 453-462, 2012.

[52] K. N. Lewis, E. Wason, Y. H. Edrey, D. M. Kristan, E. Nevoe, and R. Buffenstein, "Regulation of Nrf2 signaling and longevity in naturally long-lived rodents," Proceedings of the National Academy of Sciences of the United States of America, vol. 112, no. 12, pp. 3722-3727, 2015.

[53] R. Weindruch, R. L. Walford, S. Fligiel, and D. Guthrie, "The retardation of aging in mice by dietary restriction: longevity, cancer, immunity and lifetime energy intake," Journal of Nutrition, vol. 116, no. 4, pp. 641-654, 1986.

[54] R. J. Colman, T. M. Beasley, J. W. Kemnitz, S. C. Johnson, R. Weindruch, and R. M. Anderson, "Caloric restriction reduces 
age-related and all-cause mortality in rhesus monkeys," Nature Communications, vol. 5, article 3557, 2014.

[55] S. H. McKiernan, R. J. Colman, M. Lopez et al., "Caloric restriction delays aging-induced cellular phenotypes in rhesus monkey skeletal muscle," Experimental Gerontology, vol. 46, no. 1, pp. 23-29, 2011.

[56] L. Gross and Y. Dreyfuss, "Reduction in the incidence of radiation-induced tumors in rats after restriction of food intake," Proceedings of the National Academy of Sciences of the United States of America, vol. 81, no. 23, pp. 7596-7598, 1984.

[57] A. Bartke, M. Masternak, K. Al-Regaiey, and M. Bonkowski, "Effects of dietary restriction on the expression of insulinsignaling-related genes in long-lived mutant mice," Interdisciplinary Topics in Gerontology, vol. 35, pp. 69-82, 2007.

[58] B. P. Yu and H. Y. Chung, "Stress resistance by caloric restriction for longevity," Annals of the New York Academy of Sciences, vol. 928, pp. 39-47, 2001.

[59] D. Omodei, D. Licastro, F. Salvatore, S. D. Crosby, and L. Fontana, "Serum from humans on long term calorie restriction enhances stress resistance in cell culture," Aging, vol. 5, no. 8, pp. 599-606, 2013.

[60] S. Kannan and A. K. Jaiswal, "Low and high dose UVB regulation of transcription factor NF-E2-related factor 2," Cancer Research, vol. 66, no. 17, pp. 8421-8429, 2006.

[61] S. K. Kim and R. F. Novak, "The role of intracellular signaling in insulin-mediated regulation of drug metabolizing enzyme gene and protein expression," Pharmacology and Therapeutics, vol. 113, no. 1, pp. 88-120, 2007.

[62] B.-H. Hyun, S. S. Emerson, D.-G. Jo, M. P. Mattson, and R. De Cabo, "Calorie restriction up-regulates the plasma membrane redox system in brain cells and suppresses oxidative stress during aging," Proceedings of the National Academy of Sciences of the United States of America, vol. 103, no. 52, pp. 19908-19912, 2006.

[63] L. H. Chen, N. Hu, and D. L. Snyder, "Effects of age and dietary restriction on liver glutathione transferase activities in LobundWistar rats," Archives of Gerontology and Geriatrics, vol. 18, no. 3, pp. 191-205, 1994.

[64] A. Csiszar, T. Gautam, D. Sosnowska et al., "Caloric restriction confers persistent anti-oxidative, pro-angiogenic, and antiinflammatory effects and promotes anti-aging miRNA expression profile in cerebromicrovascular endothelial cells of aged rats," American Journal of Physiology: Heart and Circulatory Physiology, vol. 307, no. 3, pp. H292-H306, 2014.

[65] D. K. Ingram, M. Zhu, J. Mamczarz et al., "Calorie restriction mimetics: an emerging research field," Aging Cell, vol. 5, no. 2, pp. 97-108, 2006.

[66] C.-Y. Chen, J.-H. Jang, M.-H. Li, and Y.-J. Surh, "Resveratrol upregulates heme oxygenase-1 expression via activation of NFE2-related factor 2 in PC12 cells," Biochemical and Biophysical Research Communications, vol. 331, no. 4, pp. 993-1000, 2005.

[67] S. Tanigawa, M. Fujii, and D.-X. Hou, "Action of Nrf2 and Keap1 in ARE-mediated NQO1 expression by quercetin," Free Radical Biology and Medicine, vol. 42, no. 11, pp. 1690-1703, 2007.

[68] E. Balogun, M. Hoque, P. Gong et al., "Curcumin activates the haem oxygenase-1 gene via regulation of $\mathrm{Nrf2}$ and the antioxidant-responsive element," Biochemical Journal, vol. 371, no. 3, pp. 887-895, 2003.

[69] K. J. Pearson, K. N. Lewis, N. L. Price et al., "Nrf2 mediates cancer protection but not prolongevity induced by caloric restriction," Proceedings of the National Academy of Sciences of the United States of America, vol. 105, no. 7, pp. 2325-2330, 2008.
[70] S. Robida-Stubbs, K. Glover-Cutter, D. W. Lamming et al., “TOR signaling and rapamycin influence longevity by regulating SKN-1/Nrf and DAF-16/FoxO," Cell Metabolism, vol. 15, no. 5, pp. 713-724, 2012.

[71] R. A. Miller, D. E. Harrison, C. M. Astle et al., "Rapamycin, but not resveratrol or simvastatin, extends life span of genetically heterogeneous mice," The Journals of Gerontology, Series A: Biological Sciences and Medical Sciences, vol. 66, no. 2, pp. 191201, 2011.

[72] R. A. Miller, D. E. Harrison, C. M. Astle et al., "Rapamycinmediated lifespan increase in mice is dose and sex dependent and metabolically distinct from dietary restriction," Aging Cell, vol. 13, no. 3, pp. 468-477, 2014.

[73] J. E. Wilkinson, L. Burmeister, S. V. Brooks et al., "Rapamycin slows aging in mice," Aging Cell, vol. 11, no. 4, pp. 675-682, 2012.

[74] C. Lerner, A. Bitto, D. Pulliam et al., "Reduced mammalian target of rapamycin activity facilitates mitochondrial retrograde signaling and increases life span in normal human fibroblasts," Aging Cell, vol. 12, no. 6, pp. 966-977, 2013.

[75] S. F. Leiser and R. A. Miller, "Nrf2 signaling, a mechanism for cellular stress resistance in long-lived mice," Molecular and Cellular Biology, vol. 30, no. 3, pp. 871-884, 2010.

[76] A. B. Salmon, S. Murakami, A. Bartke, J. Kopchick, K. Yasumura, and R. A. Miller, "Fibroblast cell lines from young adult mice of long-lived mutant strains are resistant to multiple forms of stress," The American Journal of PhysiologyEndocrinology and Metabolism, vol. 289, no. 1, pp. E23-E29, 2005.

[77] H. Wilms, J. Sievers, U. Rickert, M. Rostami-Yazdi, U. Mrowietz, and R. Lucius, "Dimethylfumarate inhibits microglial and astrocytic inflammation by suppressing the synthesis of nitric oxide, IL-1 $\beta$, TNF- $\alpha$ and IL- 6 in an in-vitro model of brain inflammation," Journal of Neuroinflammation, vol. 7, article 30, 2010.

[78] M. J. Steinbaugh, L. Y. Sun, A. Bartke, and R. A. Miller, "Activation of genes involved in xenobiotic metabolism is a shared signature of mouse models with extended lifespan," The American Journal of Physiology - Endocrinology and Metabolism, vol. 303, no. 4, pp. E488-E495, 2012.

[79] M. Sadagurski, T. Landeryou, M. Blandino-Rosano et al., "Long-lived crowded-litter mice exhibit lasting effects on insulin sensitivity and energy homeostasis," American Journal of Physiology_Endocrinology and Metabolism, vol. 306, no. 11, pp. E1305-E1314, 2014.

[80] Y.-C. Kim, Y. Yamaguchi, N. Kondo, H. Masutani, and J. Yodoi, "Thioredoxin-dependent redox regulation of the antioxidant responsive element (ARE) in electrophile response," Oncogene, vol. 22, no. 12, pp. 1860-1865, 2003.

[81] S. Davinelli, D. C. Willcox, and G. Scapagnini, "Extending healthy ageing: nutrient sensitive pathway and centenarian population," Immunity \& Ageing, vol. 9, article 9, 2012.

[82] E. M. Mercken, B. A. Carboneau, S. M. Krzysik-Walker, and R. de Cabo, "Of mice and men: the benefits of caloric restriction, exercise, and mimetics," Ageing Research Reviews, vol. 11, no. 3, pp. 390-398, 2012.

[83] V. R. Muthusamy, S. Kannan, K. Sadhaasivam et al., "Acute exercise stress activates Nrf2/ARE signaling and promotes antioxidant mechanisms in the myocardium," Free Radical Biology and Medicine, vol. 52, no. 2, pp. 366-376, 2012.

[84] S. Zhou, Y. Wang, H. Zhe, Y. Yang, and Z. He, "Bardoxolone methyl (CDDO-Me) as a therapeutic agent: an update on 
its pharmacokinetic and pharmacodynamic properties," Drug Design, Development and Therapy, vol. 8, pp. 2075-2088, 2014.

[85] R. J. Fox, M. Kita, S. L. Cohan et al., "BG-12 (dimethyl fumarate): a review of mechanism of action, efficacy, and safety," Current Medical Research and Opinion, vol. 30, no. 2, pp. 251-262, 2014.

[86] B. Gao, A. Doan, and B. M. Hybertson, "The clinical potential of influencing Nrf2 signaling in degenerative and immunological disorders," Clinical Pharmacology: Advances and Applications, vol. 6, no. 1, pp. 19-34, 2014.

[87] C. Zhang, Z.-Y. Su, T. O. Khor, L. Shu, and A.-N. T. Kong, "Sulforaphane enhances Nrf2 expression in prostate cancer TRAMP C1 cells through epigenetic regulation," Biochemical Pharmacology, vol. 85, no. 9, pp. 1398-1404, 2013. 


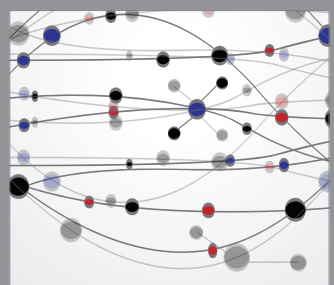

The Scientific World Journal
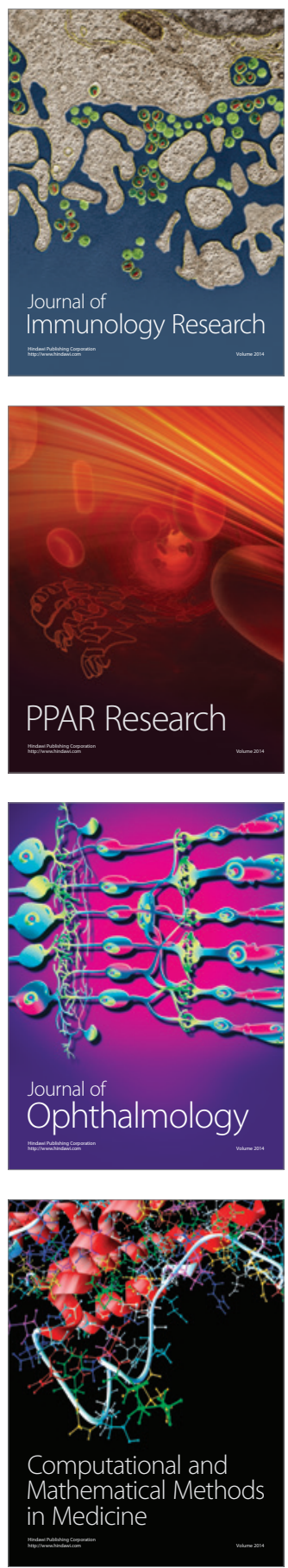

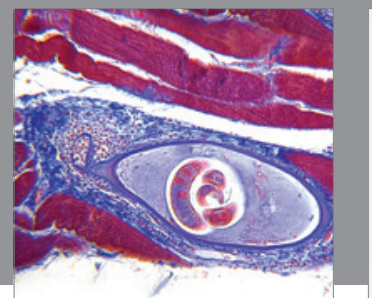

Gastroenterology

Research and Practice
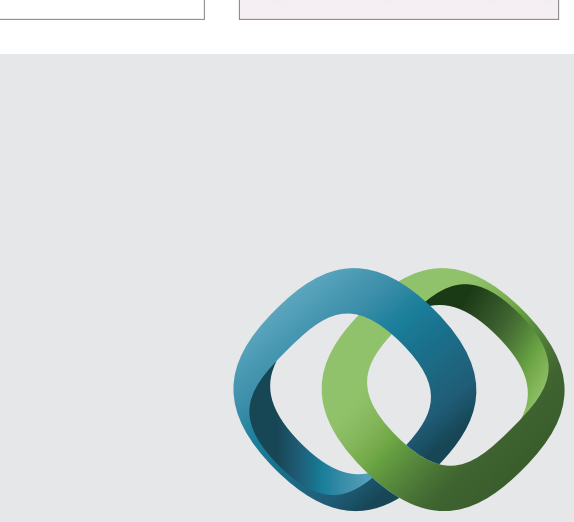

\section{Hindawi}

Submit your manuscripts at

http://www.hindawi.com
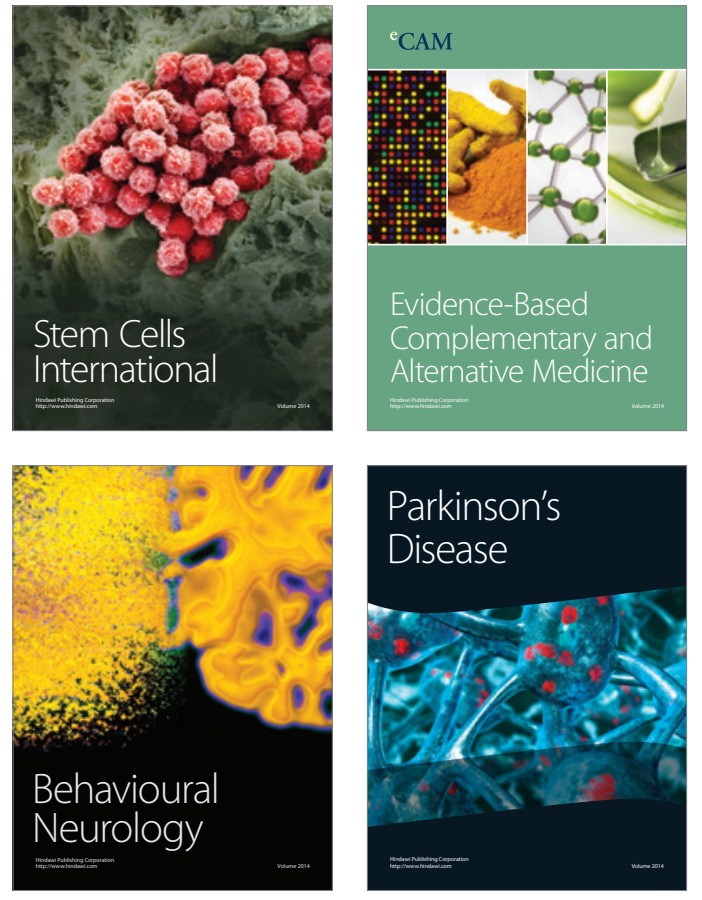
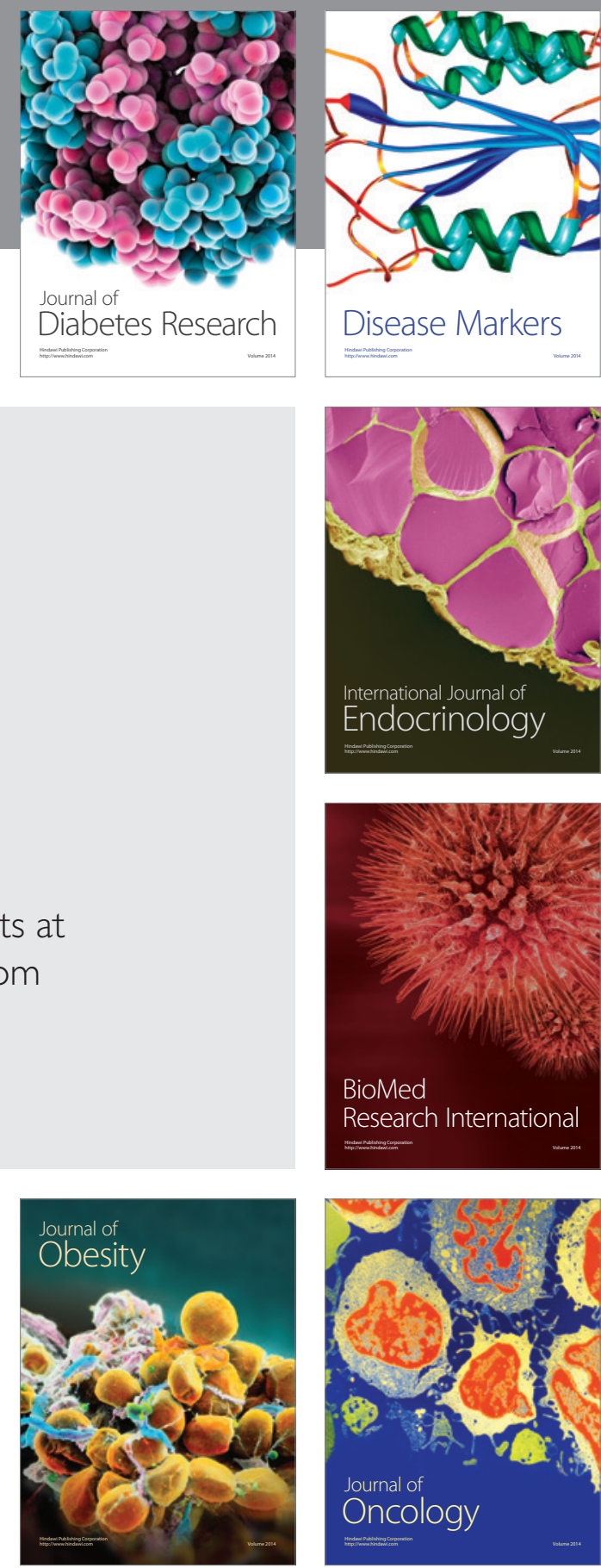

Disease Markers
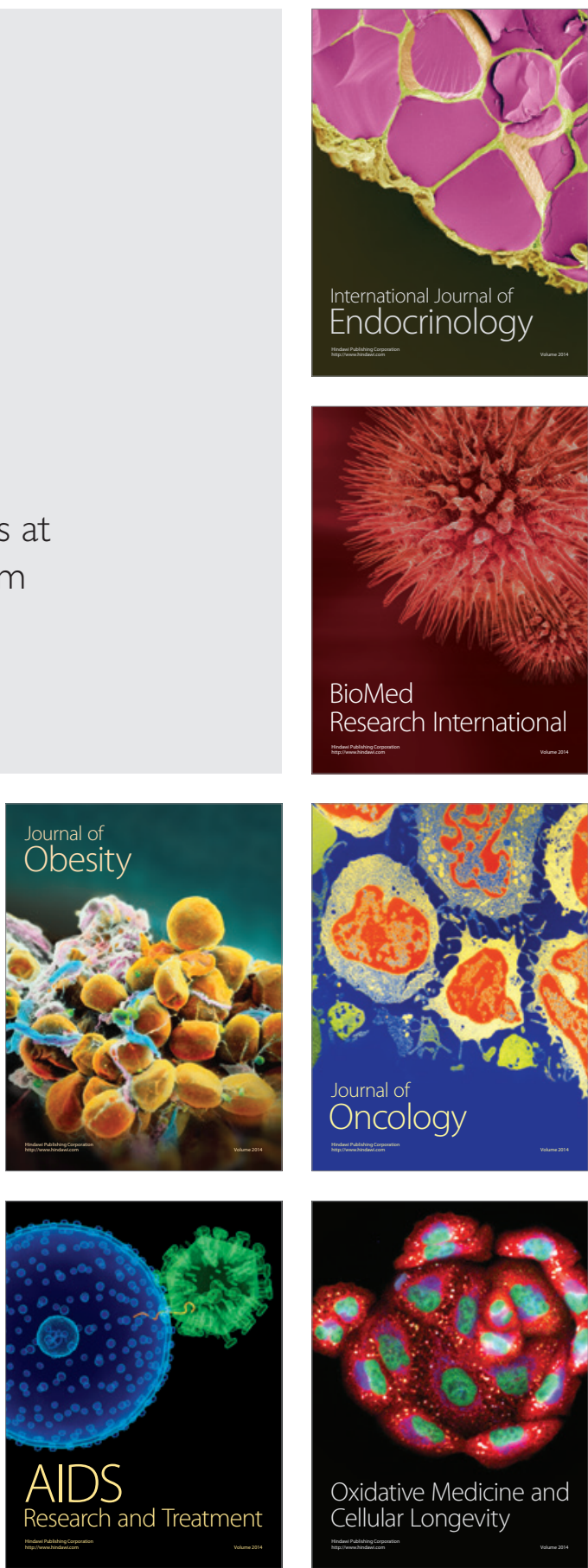